\title{
Acute His Bundle Injury during Permanent His Bundle Pacing: Mechanistic Insights into Intra- Hisian Block
}

\author{
PUGAZHENDHI VIJAYARAMAN, MD, FHRS, FACC
}

Geisinger Heart Institute, Wilkes-Barre, PA

\begin{abstract}
Permanent His bundle pacing $(H B P)$ is a physiological alternative to right ventricular pacing. Acute injury to the His bundle can occur during permanent HBP in the form of transient or permanent bundle branch blocks. Observations of mechanical injury to the His bundle during HBP lead fixation and electrophysiological response to HBP provides additional mechanistic insights into the pathology of bundle branch block and intra-Hisian atrioventricular block.
\end{abstract}

KEYWORDS. Bundle branch block, HV block, intra-Hisian block, left branch block, permanent His bundle pacing, right bundle branch block.
ISSN 2156-3977 (print) ISSN 2156-3993 (online)

(C) 2016 Innovations in Cardiac Rhythm Management

\section{Introduction}

Permanent His bundle pacing (HBP) is a physiological alternative to right ventricular (RV) pacing. RV pacing is associated with ventricular dyssynchrony, left ventricular systolic dysfunction, heart failure, and increased mortality. ${ }^{1-5}$ Despite the technical challenges during early experience with permanent $\mathrm{HBP}^{6}$ it is now clear that this form of pacing is feasible in routine clinical practice. ${ }^{7,8}$ While permanent HBP is intuitively successful in patients with atrioventricular (AV) nodal block and preserved His-Purkinje conduction, recent studies report the feasibility of HBP to correct the conduction disturbances in patients with infranodal AV block and in patients with bundle branch blocks (BBBs). ${ }^{9,10}$ Early studies in animals and humans have shown that BBBs and infranodal $\mathrm{AV}$ blocks are due to lesions within the main His bundle. ${ }^{11-13}$ This article will review the incidence of acute injury to the main His bundle during

Dr. Vijayaraman reports personal fees from Boston Scientific and personal fees from Medtronic outside the submitted work.

Manuscript received October 27, 2016, Final version accepted November 4, 2016.

Address correspondence to: Pugazhendhi Vijayaraman, MD, Director, Cardiac Electrophysiology, Program Director, CCEP fellowship, Geisinger Heart Institute, MC 36-10, 1000 E. Mountain Blvd, WilkesBarre, PA 18711. E-mail: pvijayaraman1@geisinger.edu permanent HBP and how it helps to understand the mechanisms of intra-Hisian conduction disease.

\section{His bundle injury current}

Permanent HBP utilizes the placement of an active fixation pacing lead at the His bundle region. Early in HBP experience, a traditional stylet-driven lead with retractable or exposed screw was used. ${ }^{5,14}$ HBP thresholds obtained using these leads were significantly higher $(>2 \mathrm{~V})$ than RV capture thresholds. In addition, the fluoroscopy and procedural duration for HBP were significantly longer than RV pacing because of the inability to precisely position the electrode in the His bundle region. With the availability of a dedicated pacing lead (SelectSecure $3830^{\mathrm{TM}}$, Medtronic Inc., Minneapolis, MN) and delivery sheaths, the HBP electrode can be accurately positioned in or near the His bundle region. This pacing lead is $4.1 \mathrm{Fr}$ in diameter with no inner lumen and has an exposed screw of length $1.8 \mathrm{~mm}$. The distal electrode screw is steroid eluting to allow for improved pacing thresholds. A recent study demonstrated that an acute His bundle injury current can be recorded from the unipolar or bipolar HBP lead electrograms at the time of implant in about $40 \%$ of the patients undergoing HBP (a series of 60 consecutive patients). ${ }^{15}$ In these patients the His bundle capture thresholds were significantly 
A

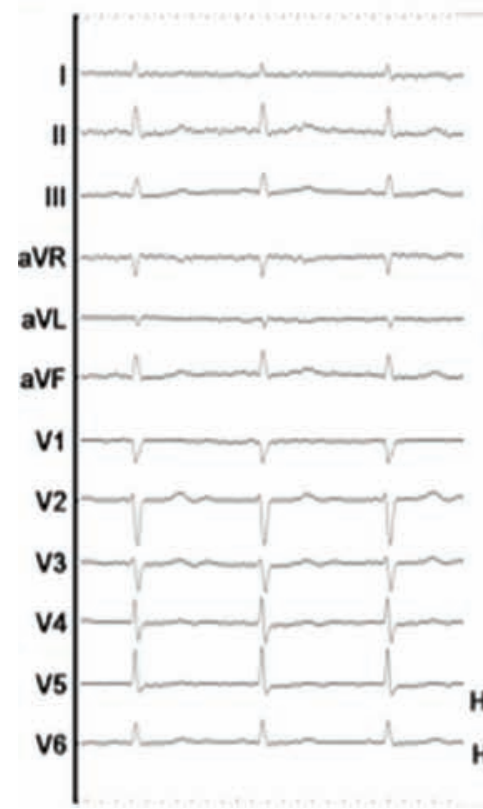

B

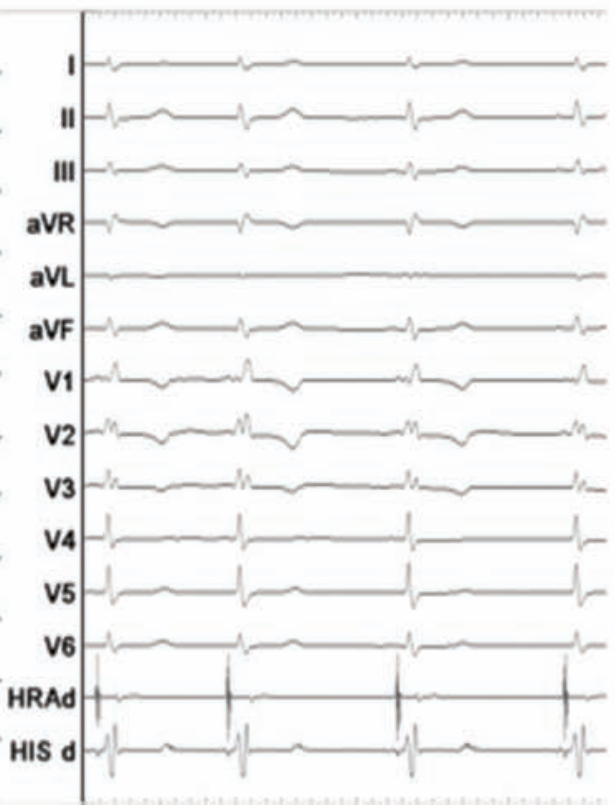

C

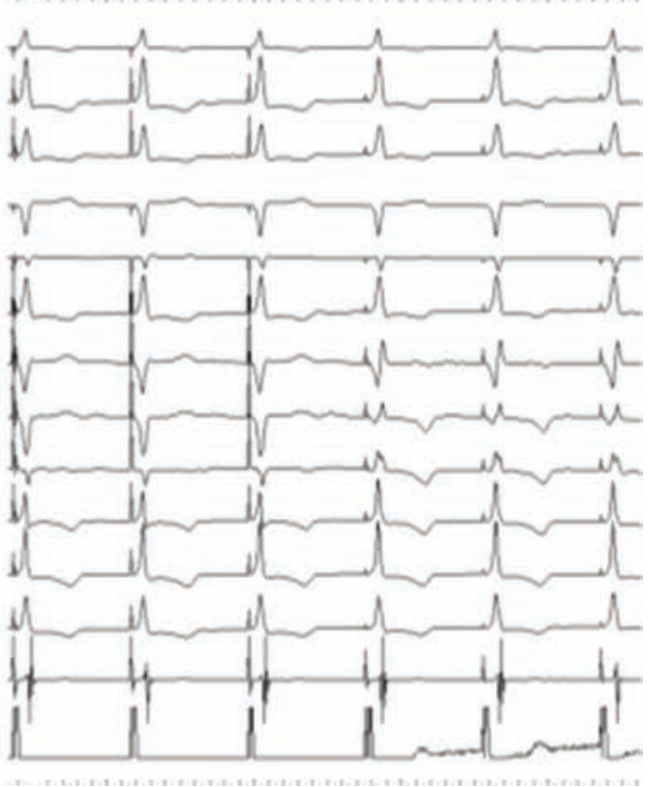

Figure 1: (a) Baseline electocardiogram with normal QRS morphology. (b) Active fixation of the His bundle pacing (HBP) lead resulted in right bundle branch block (RBBB). (c) Pacing from the HBP lead at decreasing output (from 1.5 to $1 \mathrm{~V}$ ). In the first three beats, there is non-selective HBP with correction of RBBB; In the next three beats, there is output-dependent loss of right bundle capture. RBBB resolved several minutes later.

lower than in the patients in whom His bundle injury current was not recorded. With the currently available lead and delivery systems, His bundle capture thresholds of $<1.5 \mathrm{~V}$ can consistently be achieved in the majority of patients. Recording of acute His bundle injury current during implant confirms that the lead is located directly on the His bundle and the electrode screw has penetrated the outer fibrous insulation of the His bundle itself, enabling better pacing thresholds. On the other hand, in the remaining $60 \%$ of the patients where a His bundle injury current was not recorded, it is likely that the electrode was in the vicinity of the His bundle without penetrating its fibrous sheath. It is also likely that in some patients the His bundle is deeper and could not be reached because of the short electrode screw length. Enhanced mapping techniques and leads with longer screws may help achieve more precise positioning of the HBP lead to achieve HBP thresholds similar to myocardial capture thresholds.

\section{Injury to the His bundle}

Catheter manipulation during electrophysiology study or cardiac catheterization is known to be associated with transient or permanent BBB. ${ }^{12,16}$ Targeted positioning of a permanent active fixation pacing lead in the His bundle region is expected to cause injury to the His bundle during implantation. A recent study demonstrated that during permanent HBP in 358 patients without preexisting His-Purkinje conduction disease, acute injury to the His bundle in the form of bundle branch blocks or AV block developed in $7.8 \%$ of the patients. ${ }^{17}$ RBBB developed in $5.8 \%$, LBBB in $0.8 \%$ and complete Hisventricular (HV) block occurred in $1.1 \%$ of the patients (Figures 1 and 2). The majority of these conduction disturbances occurred during active fixation of the lead, suggesting acute injury to the His bundle. In addition, when these conduction disturbances occurred during lead fixation, an injury current was invariably noted in the His bundle electrogram, confirming penetration of the electrode beyond the fibrous insulation into the His bundle itself. In $2.5 \%$ of patients, the RBBB persisted and did not resolve during follow-up. However, in the remainder of patients, the injury to the His bundle was transient with complete recovery of BBB and HV block. Development of $\mathrm{BBB}$ or complete $\mathrm{AV}$ block during permanent HBP has several clinical implications. If permanent, His bundle pacing is attempted in patients with LBBB transient or permanent RBBB could lead to complete AV block. Similarly, complete AV block can also occur in patients with normal His-Purkinje conduction. It is essential to be prepared for temporary transcutaneous or transvenous pacing. If the AV block occurred during lead fixation, pacing from the HBP lead would invariably capture the distal His bundle. Alternately, additional venous access and pacing electrode (atrial) should be readily available for transvenous pacing to avoid catastrophic consequences from prolonged asystole. Careful manipulation of the sheath with gentle movements is essential to prevent injury to the His bundle during mapping. It is preferable that mapping for the His bundle is performed from the atrial to the ventricular side of the tricuspid annulus to avoid injury to the distal His bundle early during mapping. 
A

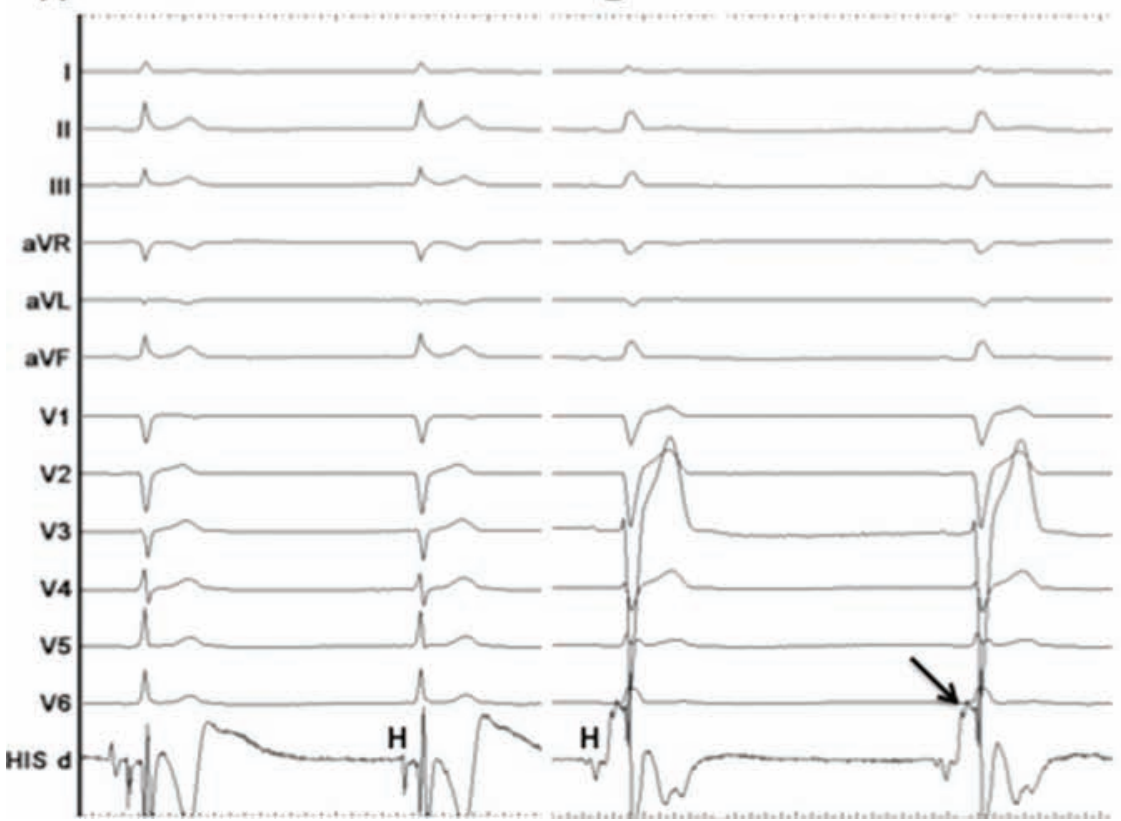

C

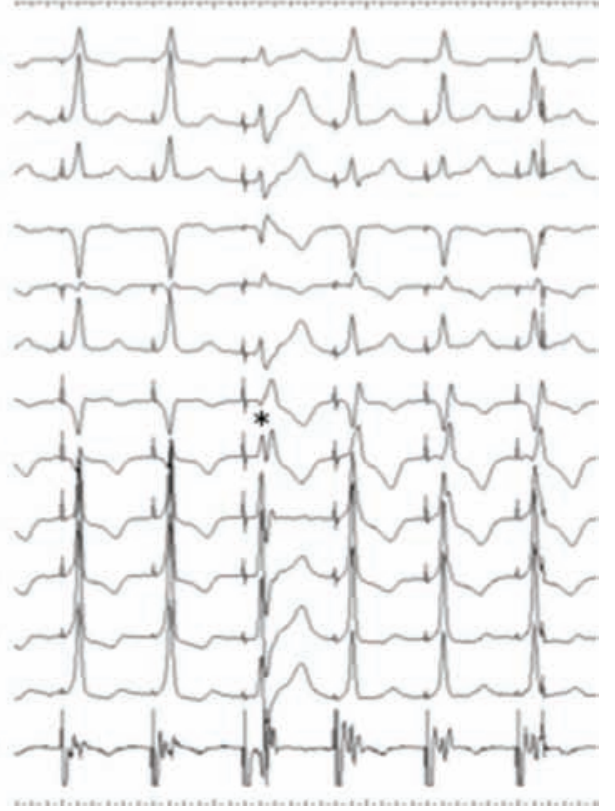

Figure 2: (a) Baseline electocardiogram with intracardiac electrogram from the His bundle pacing (HBP) lead before lead fixation. H: His. (b) Following fixation of the HBP lead, there is a significant current of injury (arrow) on the His bundle electrogram with new left bundle branch block (LBBB). (c) Pacing from the HBP lead at decreasing output (from 1.5 to $1 \mathrm{~V}$ ). The first two beats show non-selective His bundle capture with correction of LBBB. The third beat $(*)$ shows selective left bundle capture with right bundle branch block morphology. The next three beats show non-selective left bundle capture with right ventricular fusion. LBBB resolved within 30 minutes.

In addition, if the proximal His bundle is injured, it is still possible to fix the lead in the distal His bundle.

\section{Mechanistic insights}

Acute current of injury demonstrated as ST-segment elevation during myocardial lead placement is due to focally damaged cell membranes consequent to the trauma of electrode pressure against the myocardium. The resulting edema leads to an acutely higher pacing threshold of the tissue in contact but invariably improves over the next several minutes. The current of injury is a marker of excellent tissue contact and hence results in better pacing thresholds. This holds true in the setting of permanent HBP. In patients with His bundle injury current, the acute His bundle capture threshold may be high $(>2 \mathrm{~V})$ initially but then improves frequently to $<1 \mathrm{~V}$ by the end of the procedure because of resolution of the tissue edema.

Acute BBB and complete HV block that occur during HBP lead implant are most likely due to severe edema resulting from direct trauma during the lead fixation. As previously described, a His bundle injury current is recorded on the HBP lead electrograms in all these cases. ${ }^{17}$ The development of RBBB or LBBB during the lead placement in the main His bundle, correction of the induced BBB during pacing at the site, and capture of the entire His bundle during pacing confirms that discrete lesions in the main His bundle can lead to right or LBBB. Early investigators in dog models have previously demonstrated similar observations. ${ }^{18-21}$ Apart from inducing His bundle injury (intra-Hisian block) by ligating the first septal artery and causing myocardial ischemia in canine models, El-Sherif et al. ${ }^{18}$ were able to induce localized damage with a needle point inserted into the His bundle while action potentials were being recorded from microelectrodes proximal and distal to the damaged zone causing BBBs. These findings reversed after several minutes.

During permanent HBP attempts in more than 600 patients in our laboratory, we have observed six cases of complete AV block. In four of these patients there was evidence for complete HV block (Figure 3), injury current on the His bundle electrograms and idioventricular, wide-complex escape rhythms. In these patients, conduction normalized within $10 \mathrm{~min}$ to $24 \mathrm{~h}$, and His bundle capture thresholds were less than $1 \mathrm{~V}$. In two of these patients there was evidence for split His potentials and His bundle escape rhythms with narrow QRS (Figure 4). His bundle capture thresholds were acutely high $(>3 \mathrm{~V})$ and improved to less than $0.5 \mathrm{~V}$ within 24 hours. In one patient, conduction did not recover at 24 hours but had completely normalized at the 2-week follow-up. In a recent report of permanent HBP in patients with advanced AV block, in 29 of 54 (54\%) patients with infranodal disease, the His bundle could be successfully recruited by pacing at the site with evidence for $\mathrm{HV}$ block on a local electrogram recorded from the pacing lead. ${ }^{10}$ The intracardiac electrograms from the HBP lead in these patients is similar to those observed during acute 
A

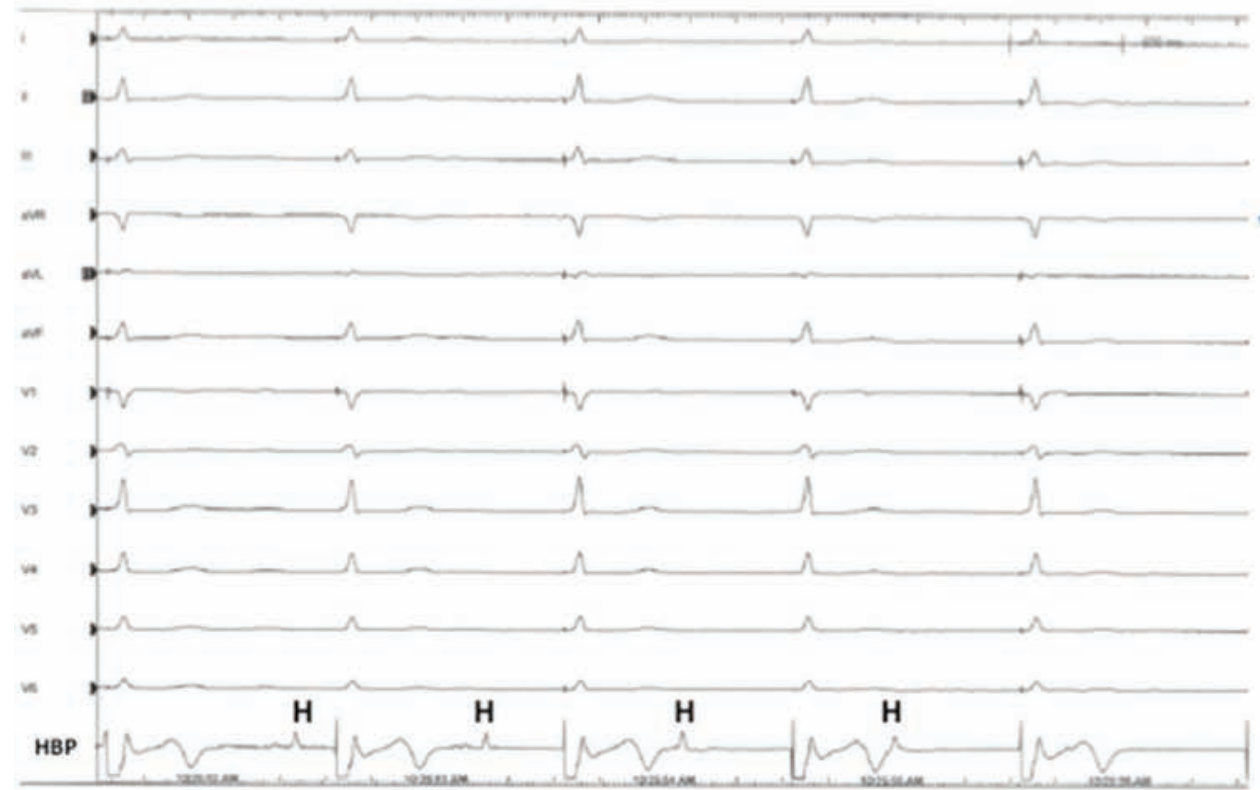

B

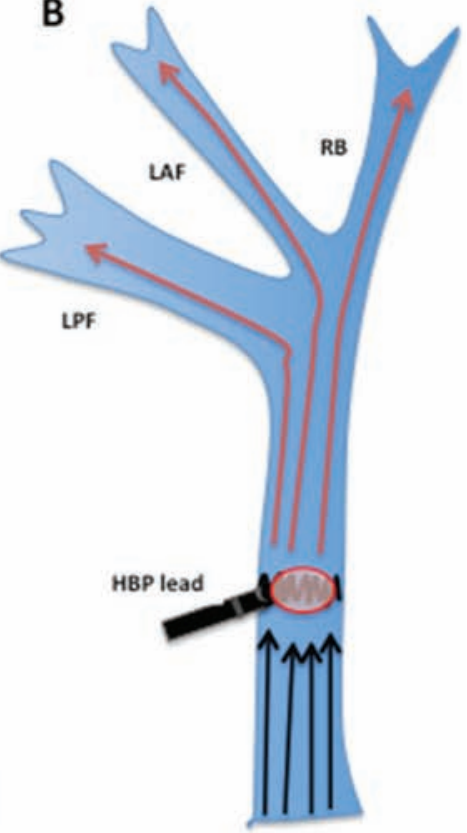

Figure 3: (a) Acute His bundle injury in the form of transient complete HV block induced during His bundle pacing lead fixation. Low output pacing (1 V) at this site results in selective His bundle capture. (b) Pictorial representation of HV block induced by the pacing lead. The intracardiac electrograms and pacing characteristics are similar to the patient shown later in Figure 5 presenting with complete HV block. The site of block and the discrete nature of the intra-Hisian lesion causing HV block is likely similar in both situations.

A



B

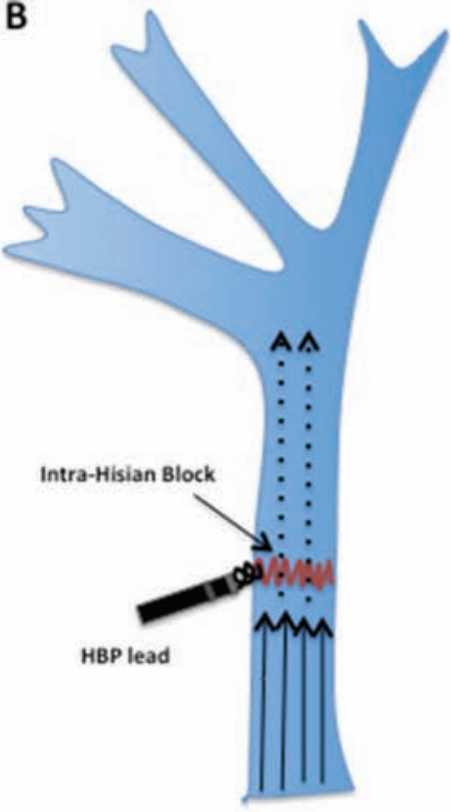

Figure 4: (a) Acute His bundle injury in the form of transient 2:1 HV block with split His signals $\left(\mathrm{H}\right.$ and $\left.\mathrm{H}^{\prime}\right)$ induced during His bundle pacing (HBP) lead implantation. This patient also demonstrated evidence for intra-Hisian Wenckebach phenomenon. (b) Pictorial representation of 2:1 intra-Hisian block induced by HBP lead fixation.

HV block induced during lead fixation (Figure 5). The ability to correct $\mathrm{HV}$ block in both these scenarios suggests that the intra-Hisian lesion causing HV block is fairly discrete, and virtual electrode polarization created during pacing is able to capture the His beyond the site of block. $^{22,23}$ In the series of HBP in infranodal AV block, 


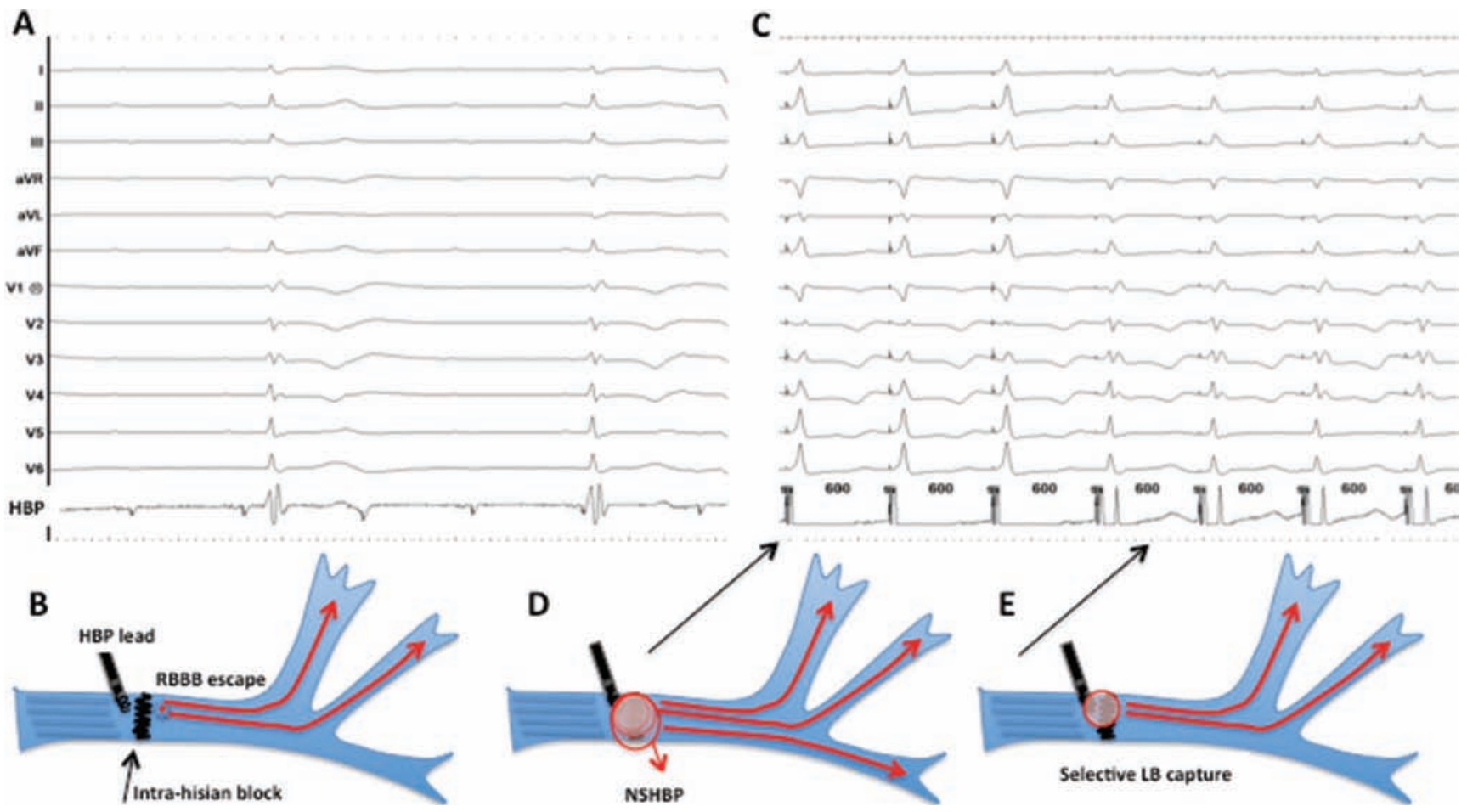

Figure 5: (a) Patient presenting with complete HV block and right bundle branch block (RBBB) escape rhythm. (b) Pictorial representation of the site of intra-Hisian AV block and presumed His bundle pacing (HBP) lead location. RBBB escape rhythm likely originates from the left bundle fibers in the main His bundle distal to the site of block. (c) During HBP at this site, there is output-dependent non-selective capture of His bundle distal to the site of block and adjacent right ventricular septal myocardium in the first three beats. In the next four beats, there is selective left bundle capture with stimulus: QRS interval of $40 \mathrm{~ms}$ and conduction with RBBB pattern similar to the escape rhythm. (d) Pictorial representation of output-dependent nonselective His bundle capture. (e) Pictorial representation of selective left bundle capture.

we reported two patients with split His potentials. A similar clinical scenario was noted during the acute HV block induced during HBP in two patients where split His potentials developed following lead fixation (Figure 4). The lesions causing intra-Hisian block with split His potentials are very discrete and pacing at these sites can overcome the conduction block. In the abovementioned series of infranodal AV block, in 12 of 54 (22\%) patients pacing at sites demonstrating HV block did not result in distal His-Purkinje conduction. Advancing the lead slightly more distally resulted in His capture and conduction. It is likely that in these patients, the intra-Hisian disease was extensive or the initial lead placement was more proximal and did not result in His capture.

During HBP lead implantation, we have also observed five cases of transient LBBB that resolved over $5 \mathrm{~min}$ to $24 \mathrm{~h}$. Pacing from the HBP lead corrected the LBBB at low outputs. Narula et al. ${ }^{11}$ had demonstrated that LBBBs are often due to longitudinal dissociation in the main His bundle with resultant asynchronous conduction delay. In addition, pacing the distal His region could correct these conduction disturbances. LBBB during HBP lead placement at the right $\mathrm{AV}$ septal region confirms these early observations. Correction of the LBBB by pacing at the site causing LBBB also attests to the discrete nature of conduction disease in the main His bundle leading to LBBB.
In the majority of patients with acute injury to the His bundle, conduction disturbances resolve. However, RBBB persisted in $2.5 \%$ of patients during follow-up. While it is conceivable that acute injury may lead to future conduction disturbances in these patients, we have not observed new $\mathrm{BBB}$ or $\mathrm{AV}$ blocks during medium-term follow-up.

\section{Conclusions}

Injury to the His bundle may occur during permanent HBP. Careful manipulation of the sheath and the lead with gentle movements is essential to prevent His bundle injury during implantation. These conduction disturbances in the form of BBB or complete HV block provide mechanistic insights into the pathophysiology of intraHisian conduction disease and offer partial explanation as to how permanent HBP can correct these conduction abnormalities. This phenomenon of His bundle injury has to be taken into consideration while designing newer leads with longer screws to improve HBP outcomes.

\section{References}

1. Lee MA, Dae MW, Langberg JL, et al. Effects of long-term right ventricular apical pacing on left ventricular perfusion, 
innervation, function and histology. I Am Coll Cardiol. 1994;24(1):225-232.

2. Tse HF, Lau CP. Long-term effect of right ventricular pacing on myocardial perfusion and function. J Am Coll Cardiol. 1997;29(4):744-749.

3. Adomian GE, Beazell J. Myofibrillar disarray produced in normal hearts by chronic electrical pacing. Am Heart J. 1986;112(1):79-83.

4. Wilkoff BL, Cook JR, Epstein AE, et al. Dual-chamber pacing or ventricular backup pacing in patients with an implantable defibrillator: The Dual Chamber and VVI Implantable Defibrillator (DAVID) Trial. JAMA. 2002;288(24): 3115-3123.

5. Sweeney MO, Hellkamp AS, Ellenbogen KA, et al. Adverse effect of ventricular pacing on heart failure and atrial fibrillation among patients with normal baseline QRS duration in a clinical trial of pacemaker therapy for sinus node dysfunction. Circulation. 2003;107(23):2932-2937.

6. Deshmukh P, Casavant D, Romanyshyn M, Anderson K. Permanent direct HB pacing: A novel approach to cardiac pacing in patients with normal His-Purkinje activation. Circulation. 2000;101(8):869-877.

7. Zanon F, Svetlich C, Occhetta E, et al. Safety and performance of a system specifically designed for selective site pacing. Pacing Clin Electrophysiol. 2011;34(3):339-347.

8. Sharma P, Dandamudi G, Naperkowski A, et al. Permanent His bundle pacing is feasible, safe and superior to right ventricular pacing in routine clinical practice. Heart Rhythm. 2015;12(2):305-312.

9. Barba-Pichardo R, Moriña-Vázquez $P$, Fernández-Gómez JM, Venegas-Gamero J, Herrera-Carranza M. Permanent His-bundle pacing: seeking physiological ventricular pacing. Europace. 2010;12(4):527-533.

10. Vijayaraman P, Naperkowski A, Ellenbogen KA, Dandamudi G. Electrophysiologic insights into site of atrioventricular block. Lessons from permanent his bundle pacing. JACC Clin Electrophysiol. 2015;1(6):571-581.

11. Narula O. Longitudinal dissociation in the His bundle. Bundle branch block due to asynchronous conduction within the His bundle in man. Circulation. 1977;56(6): 996-1006.

12. Akhtar M, Danato AN, Gilbert-Leeds CJ, et al. Induction of iatrogenic electrocardiographic patterns during electrophysiologic studies. Circulation. 1977;56(1):60-65.
13. El-Sherif N, Amay-Y-Leon F, Schonfield C, et al. Normalization of bundle branch block patterns by distal His bundle pacing. Clinical and experimental evidence of longitudinal dissociation in the pathologic his bundle. Circulation. 1978; 57(3):473-483.

14. Occhetta E, Bortnik M, Magnani A, et al. Prevention of ventricular desynchronization by permanent para-hisian pacing after atrioventricular node ablation in chronic atrial fibrillation: a crossover, blinded randomized study versus right ventricular pacing. J Am Coll Cardiol. 2006;47(10): 1938-1945.

15. Vijayaraman P, Dandamudi G, Worsnick SA, Ellenbogen KA. Acute His-Bundle injury current during permanent His-Bundle pacing predicts excellent pacing outcomes. Pacing Clin Electrophysiol. 2015;38(5):540-546.

16. Murasato $Y$, Ninomiya $K$, Imai $M$, et al. Complete atrioventricular block during left heart catheterization. Jpn Circ J. 1994;58(8):671-675.

17. Vijayaraman P, Dandamudi G, Ellenbogen KA. Electrophysiological Observations of Acute His bundle Injury during Permanent His Bundle Pacing. I Electrocardiol. 2016;49(5):664-669.

18. El-Sherif N, Scherlag BJ, Lazzara R. Conduction disorders in the canine proximal His-Purkinje system following acute myocardial ischemia. I. The pathophysiology of intra-His bundle block. Circulation. 1974;49(5):837-847.

19. Lazzara R, Yeh BK, Samet P. Functional transverse interconnections within the His bundle and the bundle branches. Circ Res. 1973;32(4):509-515.

20. El-Sherif N, Scherlag BJ, Lazzara R. Conduction disorders in the canine proximal His-Purkinje system following acute myocardial ischemia. II. The pathophysiology of bilateral bundle branch block. Circulation. 1974;49(5):848-857.

21. Scherlag BJ, El-Sherif N, Hope RR, Lazzara R. The significance of dissociation of conduction in the canine His bundle. Electrophysiological studies in vivo and in vitro. J Electrocardiol. 1978;11(4):343-354.

22. Sambelashvili AT, Nikolski VP, Efimov IR. Virtual electrode theory explains pacing threshold increase caused by cardiac tissue damage. Am J Physiol Heart Circ Physiol. 2004;286(6): H2183-2194.

23. Cheng Y, Mowrey KA, Van Wagoner DR, Tchou PJ, Efimov IR. Virtual electrode-induced reexcitation: A mechanism of defibrillation. Circ Res. 1999;85(11):1056-1066. 